\title{
Torsional And Lateral Eigenmode Oscillations For Atomic Resolution Imaging Of HOPG In Air Under Ambient Conditions
}

\section{Anna L. Eichhorn}

Technische Universität Darmstadt

Christian Dietz ( $\sim$ dietz@pos.tu-darmstadt.de )

Technische Universität Darmstadt

\section{Research Article}

Keywords:

Posted Date: February 17th, 2022

DOI: https://doi.org/10.21203/rs.3.rs-1320660/v1

License: (c) (1) This work is licensed under a Creative Commons Attribution 4.0 International License.

Read Full License 


\title{
Torsional and lateral eigenmode oscillations for atomic
}

\section{resolution imaging of HOPG in air under ambient}

\author{
conditions
}

\author{
Anna L. Eichhorn ${ }^{1}$ and Christian Dietz ${ }^{1 *}$ \\ ${ }^{1}$ Physics of Surfaces, Institute of Materials Science, Technische Universität Darmstadt, \\ Alarich-Weiss-Str. 2, 64287 Darmstadt, Germany \\ Email: eichhorn@pos.tu-darmstadt.de,dietz@pos.tu-darmstadt.de
}

ORCID-IDs: A.L. Eichhorn: 0000-0003-1754-0276, C. Dietz: 0000-0002-4134-7516

\begin{abstract}
Combined in-plane and out-of-plane multifrequency atomic force microscopy techniques have been demonstrated to be important tools to decipher spatial differences of sample surfaces at the atomic scale. The analysis of physical properties perpendicular to the sample surface is routinely achieved from flexural cantilever oscillations, whereas the interpretation of in-plane sample properties via force microscopy is still challenging. Besides the torsional oscillation, there is the additional option to exploit the lateral oscillation of the cantilever for in-plane surface analysis. In this study, we used different multifrequency force microscopy approaches to attain better understanding of the interactions between a super-sharp tip and an HOPG surface focusing on the discrimination between friction and shear forces. We found that the lateral eigenmode is suitable for the determination of the shear modulus whereas the torsional eigenmode provides information on local friction forces between tip and sample. Based on the results, we propose that the full set of elastic constants of graphite can be determined from
\end{abstract}


combined in-plane and out-of-plane multifrequency atomic force microscopy if ultrasmall amplitudes and high force constants are used.

\section{Introduction}

Understanding the in-plane nanomechanical behavior of graphitic surfaces at the atomic scale under ambient conditions in air is of utmost importance to predict the long-term performance of graphene-based nanodevices such as van der Waals heterostructures ${ }^{1}$ or for DNA sequencing ${ }^{2}$. Multifrequency atomic force microscopy (AFM) $)^{3}$ was shown to be an excellent tool for the quantification of forces at the nanoscale in out-of-plane as well as in in-plane direction $^{4-6}$. The out-of-plane force deconvolution and determination of elastic moduli from bimodal AFM spectroscopy data using the Sader method ${ }^{7}$ or the matrix method ${ }^{8}$ are well established. In contrast, the determination of in-plane forces and shear moduli from spectroscopic data is still challenging ${ }^{9,10}$. One issue is the difficulty to excite the cantilever in the in-plane direction. This problem can be addressed by using photothermal excitation ${ }^{11,12}$. The technique is based on a power-modulated laser focused at the base of the cantilever and a few micrometers off the cantilever length symmetry axis. This results in an in-plane oscillation if the excitation frequency matches the resonance frequency of the desired eigenmode. Photothermal excitation facilitated atomic resolution imaging using the torsional-eigenmode oscillation in bimodal $\mathrm{AFM}^{13}$. A second issue is the discrimination between torsional and lateral eigenmodes. In most publications the term "lateral" is used as synonym for both, the torsional and lateral eigenmode. This might originate from the fact that in theory the lateral-eigenmode oscillation should not be observable using the beam detection methods as schematically explained by Ding et al. ${ }^{14}$. However, they rationalized that the tip attached to the cantilever can induce coupling between lateral and torsional eigenmodes facilitating the detection of lateral oscillations using a standard detection laser and segmented photodiodes. The authors demonstrated that both eigenmodes are useful for imaging and that the lateral oscillation might 
be advantageous over the torsional one. The main issue in using the lateral eigenmode for imaging is the calibration of the inverse optical lever sensitivity (invOLS) to attain quantitative or semi-quantitative data. There are a few approaches for the determination of the torsional invOLS ${ }^{15-18}$, whereas there is currently no procedure for the determination of the lateral invOLS to the best of our knowledge.

In this study, we compared the suitability of the torsional and the lateral eigenmodes for atomic resolution imaging of HOPG in air under ambient conditions. The comparison is based on our recently published methodology named AMFlex2-FMTor1-FMFlex3 mode introduced in Ref. [4] with a slightly modified setup (AMFlex2-FMLat1-FMFlex3). Both setups exploit the second flexural eigenmode for the topographical feedback in amplitude modulation (AM) whereas the first torsional, first lateral and third flexural mode are frequency-modulated (FM) and controlled by phase-locked-loops (PLL) electronics. Inspired by the work of colleagues in the field, we used higher flexural eigenmodes ${ }^{19,20}$ and small free amplitudes in combination with small amplitude setpoints for imaging ${ }^{21-23}$.

For some of the super-sharp tips we used, it was impossible to excite the second flexural and the first torsional eigenmode individually due to the close proximity of their resonance frequencies. Interestingly, the coupling of the two eigenmodes facilitated atomic resolution imaging. A scheme of possible setups as well as the resulting movement of the tip are schematically shown in Figure 1. If there is coupling between the second flexural and the first torsional eigenmode, the torsional oscillation cannot be controlled individually (open loop $(\mathrm{OL})$ ). From the second flexural and the first torsional phase shifts, dissipation in out-of-plane and in-plane direction can be determined, respectively. Unfortunately, the AMFlex2-OLTor1 mode is not suitable for the deconvolution of in-plane or out-of-plane forces. Consequently, we extended the AMFlex2-OLTor1 mode, by two phase-locked loops (PLL) for frequency modulation (FM) of the first lateral and the third flexural eigenmode. The resulting AMFlex2- 
OLTor1-FMLat1-FMFlex3 mode such as schematically depicted in Figure 1 facilitates quantification of in-plane and out-of-plane forces, reconstructed from lateral- and thirdeigenmode flexural frequency-shift data.

In this work, we analyzed the imaging capability of coupled flexural/torsional oscillations as well as the suitability of the first lateral eigenmode for atomic resolution imaging and provide an approach for the calibration of the lateral eigenmode optical lever sensitivity. In addition, we calculated in-plane forces from torsional and lateral frequency-shift data and analyzed the origin of the forces regarding friction or shear. Analyzing the tip-trajectories, effective torsional and lateral amplitudes as well as the maximum indentation of the tip into the surface (with and without compression) were estimated. The plausibility of the determined lateral forces was confirmed by calculating the lateral displacement using the literature value of the shear modulus of HOPG. 


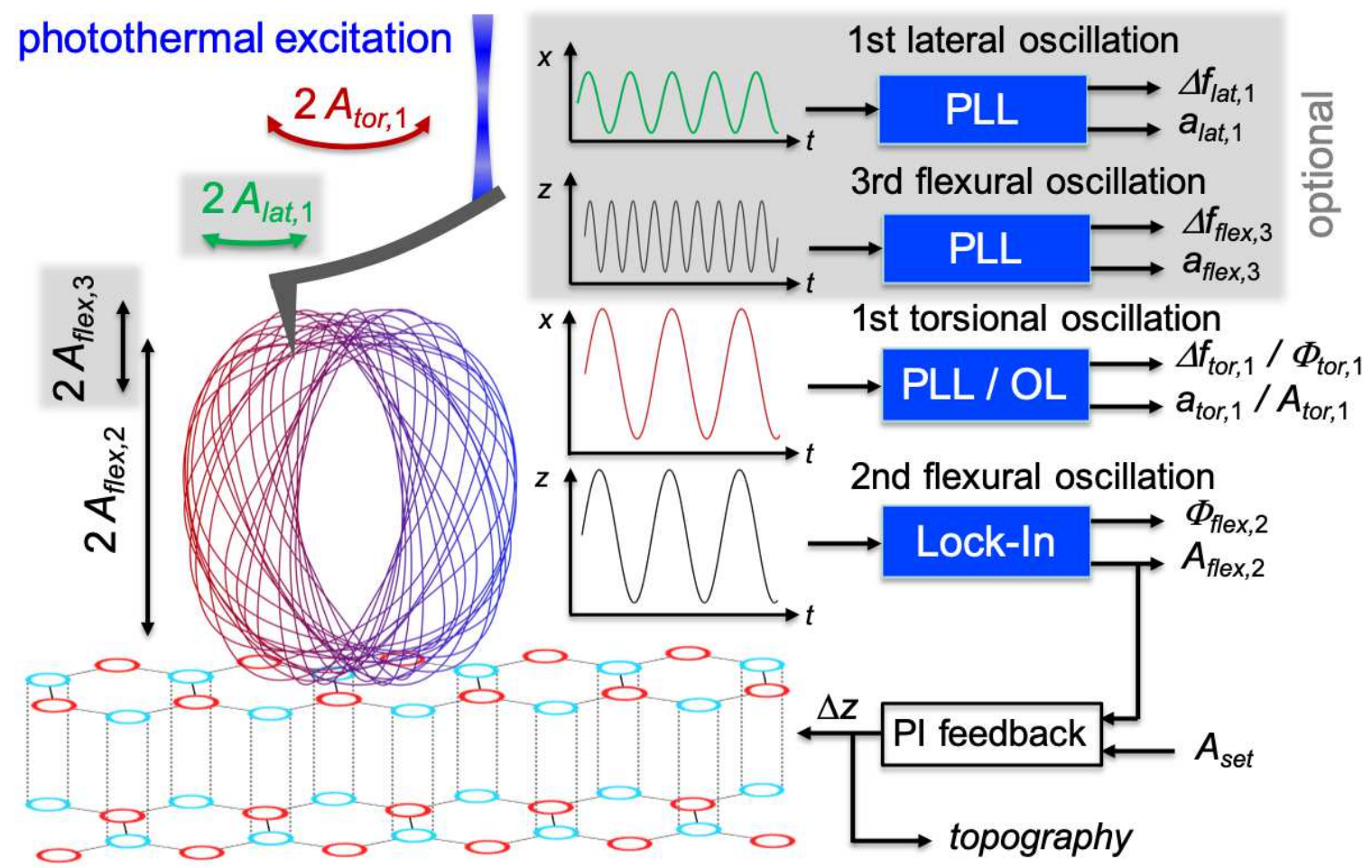

Figure 1: Schematic illustration depicting the setup of the AMFlex2-OLTor1-FMLat1FMFlex3 mode. Topographical feedback is driven at the coupled resonance frequency of the second flexural and the first torsional eigenmode using the flexural component for amplitude modulation. The first-torsional-eigenmode amplitude and phase are recorded at the same frequency in an open-loop configuration. Optionally, the setup can be expanded by one or two phase-locked loops, tracking the phase at resonance of the first lateral and/or the third flexural eigenmode which allowed us to quantify forces in in-plane and/or out-of-plane direction from the respective frequency-shift data. Dissipative tip-sample interactions can be analyzed from the drive amplitudes $\left(a_{l a t, 1}, a_{f l e x, 3}\right)$ and the respective phase shifts $\left(\Phi_{f l e x, 2}, \Phi_{t o r, 1}\right)$. Note: The cantilever dimension was substantially reduced for simplicity. 


\section{Results and Discussion}

\section{Imaging capability of the AMFlex2-OLTor1-FMLat1 mode for atomic resolution}

From Figure 2, we get an overview of the capability of different imaging channels for atomic resolution imaging in air under ambient conditions depending on the $z$-sensor position. Increasing $z$-sensor position (from left to right) causes a reduction of the average tip-sample distance. Figure 2a-e show schemes of the expected tip-trajectory corresponding to the AMFlex2-OLTor1-FMLat1 mode with a lateral-eigenmode amplitude setpoint of $A_{\text {lat }, 1}=863 \mathrm{pm}$. The shown tip-trajectories were calculated by plotting the out-of-plane deflection $z(t) v s$. the in-plane deflection $x(t)$ of the cantilever using linear combinations of cosine functions such as shown by Benaglia et al. ${ }^{24}$ for the $z$-component (see eqs. (S1) and (S2) of the Supplementary Information for details). The lateral-eigenmode invOLS was calibrated at a wrinkle of a graphene layer on an HOPG sample as explained in detail in section 2 (Calibration of lateral-oscillation-eigenmode sensitivity). From Figure 2a-e the second flexuraleigenmode-amplitude setpoint is reduced from $700 \mathrm{pm}$ to $454 \mathrm{pm}$, causing a reduction of the torsional amplitude due to the coupling of both modes (Fig. 2, second row). The corresponding approximate $z$-sensor position can be taken from the third row of Figure 2. From spectroscopic experiments (local amplitude distance curves recording all available observables), we can visualize the dependence of the second flexural amplitude (Fig. 2f), the first torsional amplitude (Fig. 2l) and the first lateral frequency shift (Fig. 2r) on the $z$-sensor position. The crossed circles mark the positions where the AFM images of Figure 2 where taken. The height images are shown in Figure 2g-k, the "error images" of the first torsional amplitude in Figure 2m-q and the first lateral frequency-shift images in Figure 2s-w. "Error images" of the first torsional amplitude here means the deviation of the actual torsional amplitude relative to the torsionalamplitude setpoint corresponding to the second flexural-amplitude setpoint due to the coupling of both eigenmodes. 


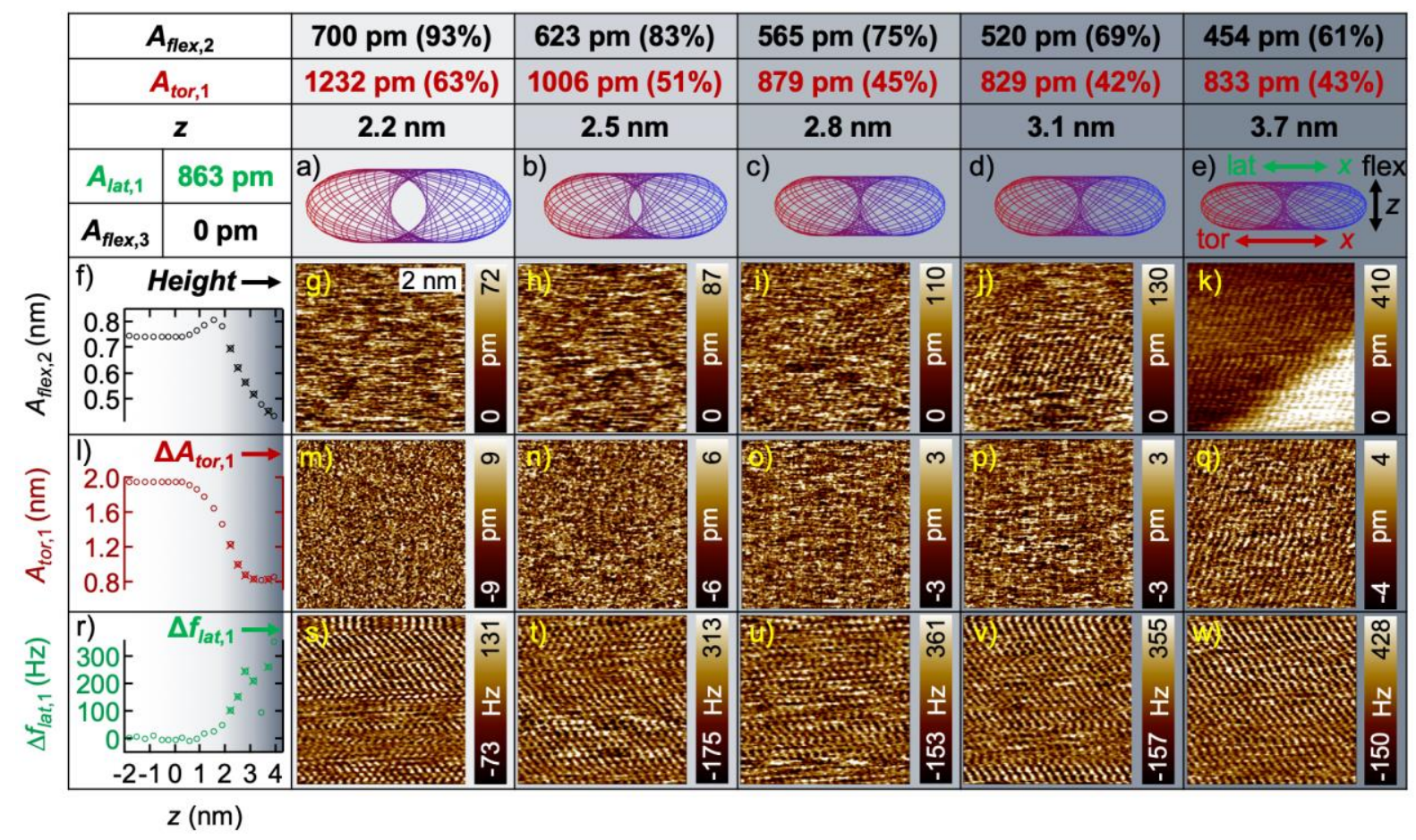

Figure 2: Imaging and spectroscopy on HOPG at a covered step edge in AMFlex2-OLTor1FMLat1 mode with 863 pm lateral-eigenmode-amplitude setpoint. (a-e) Schemes of the tip trajectory for the coupled second flexural and first torsional cantilever oscillation for decreasing second flexural-eigenmode-amplitude setpoints $A_{f l e x, 2 .}$ The setpoints of $A_{f l e x, 2}$ are listed in the first, the corresponding setpoints of the first-torsional-eigenmode amplitude $A_{t o r, 1}$ in the second row and the approximate $z$-sensor position at the third row. The values in brackets represent the

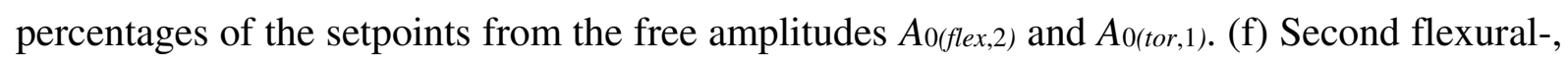
(l) first torsional-eigenmode amplitude $A_{t o r, 1}$ and (r) first lateral frequency shift $\Delta f_{\text {lat }, 1} v s . z^{-}$ sensor position, where the crossed circles mark the positions at which the images where taken. (g-k) Height, (m-q) first torsional-eigenmode amplitude error $\Delta A_{t o r, 1}$ and (s-w) $\Delta f_{\text {lat }, 1}$ images at decreasing Aflex,2.

Comparing the amplitude vs. z-position curves in Figure $2 \mathrm{f}$ and 1 , it becomes evident that although the second flexural and the first torsional oscillations are coupled, they show different dependencies on the tip-sample distance. These differences are consistent with results from uncoupled flexural-torsional AFM studies in open-loop configuration ${ }^{13}$. The lateral frequency 
shift vs. $z$-sensor position curve in Figure 2r shows an increasing repulsive interaction with decreasing tip-sample distance. Atomic resolution imaging was feasible in all three channels as e.g. visible in Figure $2 \mathrm{k}, \mathrm{q}$ and $\mathrm{w}$ at a $z$-sensor position of approximately $3.7 \mathrm{~nm}$. Strikingly, the lateral frequency-shift images show atomically resolved structures at setpoint ratios up to $93 \%$ of the second flexural-eigenmode amplitude as observable in Figure 2s, which corresponds to a $z$-sensor value of approximately $2.2 \mathrm{~nm}$. Note that these imaging conditions reflect gentle tipsample interaction preserving the integrity of both tip and sample. At this position, however, neither in the height image (Fig. 2g) nor in the torsional-amplitude error image (Fig. 2m) atomic contrast could be resolved. Lowering the second flexural-eigenmode-amplitude setpoint, atomic contrast becomes more and more apparent in the height images (Figure $2 \mathrm{~m}-\mathrm{q}$ ). The growing corrugation amplitude (measured vertical distance between atomic and hollow side) is assumed to originate from an increase in the mean cantilever deflection ${ }^{13,25,26}$. Strikingly, the height image at a $z$-sensor position of $3.7 \mathrm{~nm}$ (Fig. 2k) shows a step which matches the height of individual graphene layers. This step is neither observable in the $\Delta A_{t o r, 1}$ image (Fig. 2q) nor in the $\Delta f_{\text {lat }, 1}$ image at the same $z$-sensor position. Consequently, the topographic feedback seems to function extremely precise. Another interesting observation is, that the atomic structure at both, the lower and the upper plateau of the graphene step, exhibit the same arrangement of carbon atoms. This leads us to the conclusion that we imaged a monoatomic step edge covered by a small number of graphene layers which is in line with results shown by Abooalizadeh et $a l .{ }^{27}$. From this observation the question arises why we were not able to resolve the step at smaller $z$-sensor positions. To answer the question, we exemplarily drew two cross sectional profiles through the height images shown in Figure $2 \mathrm{k}$ and $\mathrm{j}$ (see Figure $\mathrm{S} 1$ in the Supplementary Information). We assume that the visibility of the step edge depends on whether the average tip-sample force (represented by the mean deflection, averaged over several oscillation cycles) is predominantly attractive or repulsive. On the right side of Figure S1, we schematically sketched the alleged interaction between tip and sample on a covered step edge. In the 
predominantly attractive regime, we assume that several graphene layers cover the step such as depicted in the top right part of Figure S1. As discussed in our recent work and by others ${ }^{4,28}$, it is reasonable to believe that the topmost carbon layers are lifted by the attractive tip-sample interactions. Consequently, the tip will not sense the step edge. If imaging takes place in the predominantly repulsive regime, we assume that the graphene cover layers are tightly stretched over the step edge as sketched in the bottom right of Figure S1.

From the imaging results shown in Figure 2 we can conclude that the coupled motion of the cantilever (AMFlex2-OLTor1) seems to promote atomic resolution at higher setpoint ratios $\left(A_{f l e x, 2} / A_{0}(f l e x, 2)\right)$ compared to uncoupled ones. This can, on the one hand, be attributed to the enhanced dynamic stiffness of the coupled flexural/torsional oscillation of the cantilever and, on the other hand, to the oval shape of the tip trajectory. This might lead to a reduced influence of the interactions far away from the center of the oscillation, because the tip will have the closest distance to the sample and therefore the highest interaction at the currently measured local position. Nevertheless, the largest drawback of imaging with coupled cantilever modes is that the relation between the flexural and the torsional amplitudes cannot be set individually. Additionally, it needs to be considered that the combined in-plane oscillation of torsional and lateral components needs to be analyzed in detail in order to get a better understanding regarding the interaction with the sample. 


\section{Calibration of lateral oscillation amplitude inverse optical lever sensitivity}

The lateral cantilever oscillation is rarely used for AFM imaging because of the difficulties to calibrate its inverse optical lever sensitivity (invOLS). As discussed in detail by Ding et al. ${ }^{14}$ the lateral oscillation itself cannot be detected by the photodiode. But due to the tip attached to the cantilever, a small torsion is induced which results in a measurable movement of the reflected laser beam on the photodiode. While the torsional oscillation amplitude invOLS can be determined e.g. from thermal noise spectra ${ }^{15}$, there is to the best of our knowledge currently no method for the lateral oscillation invOLS calibration. Here, we present an approach based on imaging a graphene wrinkle on an HOPG sample with different lateral-oscillation-amplitude setpoints in AMFlex2-OLTor1-FMLat1 mode. The idea behind this method is, that the effect of an increasing lateral oscillation amplitude can be compared to a smoothening of imaging data points. In Figure $3 \mathrm{a}$ and $\mathrm{f}$ we show the averaged cross sections (20 pixel) through the height images (b-e) and the lateral frequency-shift images (g-j) taken at different lateral-oscillationamplitude setpoints. All images were taken at a constant second flexural-eigenmode amplitude of $238 \mathrm{pm}$ and at a scan angle of $90^{\circ}$ resulting in both, fast scan direction and lateral oscillation direction being perpendicular to the graphene wrinkle. The orange $(b, g) / b l a c k(c, h) / b l u e$ $(\mathrm{d}, \mathrm{i}) / \mathrm{red}(\mathrm{e}, \mathrm{j})$ marked cross sections result from the imaging data taken at lateral-oscillationamplitude setpoints of $5 \mathrm{mV} / 10 \mathrm{mV} / 20 \mathrm{mV} / 40 \mathrm{mV}$. The black/blue/red dotted lines in Figure $3 \mathrm{a}$ and $\mathrm{f}$ result from smoothening of the orange curves $($ Alat, $1=5 \mathrm{mV})$ over $23 / 45 / 89$ pixel and multiplication (height)/division $\left(\Delta f_{\text {lat }, 1}\right)$ by the weighing factors $1.25 / 1.85 / 2.80$ (details are given in the Supplementary Information). 


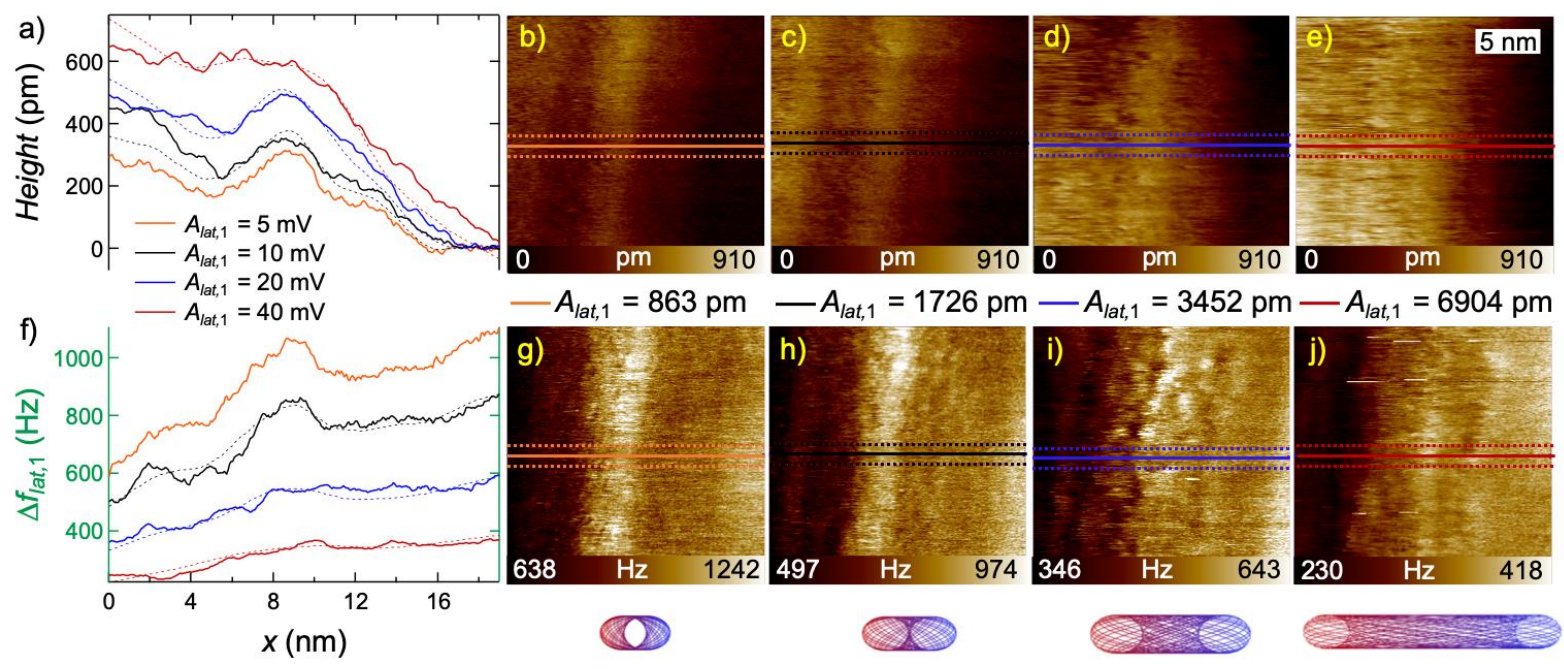

Figure 3: Calibration of lateral-oscillation-eigenmode amplitude at a graphene wrinkle on HOPG. The solid lines in a) and f) show the height and the lateral frequency shift for different lateral-eigenmode setpoints along the averaged cross sections (20 pixel) marked in b-e) and gj). The images were taken in the AMFlex2-OLTor1-FMLat1 mode with a free second flexuraleigenmode amplitude of $765 \mathrm{pm}$ and a setpoint of $238 \mathrm{pm}$. The scan angle was $90^{\circ}$ so that both, the fast scan direction and the direction of the lateral oscillation were orineted perpendicular to the wrinkle. The dotted black/blue/red lines in a) and f) represent the orange curve smoothed over 23/45/89 pixel and multiplication (height)/division $\left(\Delta f_{\text {lat }, 1}\right)$ by the weighing factors 1.25/1.85/2.80 (see Supplementary Information for details). The alleged tip-trajectories are drawn schematically at the bottom reight.

From the cross sections in Figure $3 \mathrm{a}$ and $\mathrm{f}$ and from the corresponding images in Figure $3 \mathrm{~b}-\mathrm{e}$

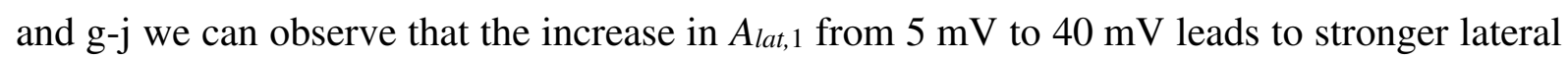
averaging of the height and the lateral frequency-shift values. If we compare e.g. the orange $\left(A_{\text {lat }, 1}=5 \mathrm{mV}\right)$ with the red curve $\left(A_{\text {lat }, 1}=40 \mathrm{mV}\right)$ in Figure $3 \mathrm{a}$ we can directly see that for the smaller $A_{\text {lat }, 1}$ two hillocks can be resolved whereas for the larger $A_{\text {lat }, 1}$ only one broader hillock is visible. In Figure $3 \mathrm{f}$ the lateral frequency shift shows a clearly visible hillock for $A_{\text {lat }, 1}=5 \mathrm{mV}$ (orange) whereas for $A_{\text {lat }, 1}=40 \mathrm{mV}$ (red) the hillock is hardly visible. Based on these 
observations we assumed that the cross sections of the images taken with $A_{\text {lat }, 1}=10 \mathrm{mV}$, $A_{l a t, 1}=20 \mathrm{mV}$ and $A_{l a t, 1}=40 \mathrm{mV}$ can be reproduced by smoothening of the orange curve which represents the cross sectional data resulting from $A_{\text {lat }, 1}=5 \mathrm{mV}$. By smoothening the orange curves in Figure 3a and f with a Savitzky-Golay filter of first grade over 23, 45 and 89 pixel, respectively, we were able to reproduce the general trend of the black, blue and red curves with satisfying accuracy. After treating the smoothed averaged cross-sections with appropriate weighing factors (see Supplementary Information for details) the black, blue and red dotted curves shown in Figure 3a and f were gained. Although there are small deviations between the dotted and the solid curves in Figure 3a, in particular at the end and the beginning of the cross sections, the overall trend is nicely reproduced. From the number of pixel over which the smoothening was performed, we determined the optical invOLS of the lateral oscillation amplitude according to equations $(2,3)$

$$
\begin{gathered}
\left(A_{\text {lat }, 1}-A_{\text {lat }, 1(\text { ref })}\right)[\mathrm{nm}]=\frac{l}{n_{\text {line }}} \frac{n_{\text {smooth }}-1}{2}, \\
s_{\text {lat }, 1}=\frac{\left(A_{\text {lat }, 1}-A_{\text {lat }, 1(\text { ref })}\right)[\mathrm{nm}]}{\left(A_{\text {lat }, 1}-A_{\text {lat }, 1(\text { ref })}\right)[\mathrm{V}]}
\end{gathered}
$$

where $A_{\text {lat } 1 \text { (ref) }}$ is the lateral-amplitude setpoint which was used for the reference image and the corresponding cross section chosen to be smoothened (here: $A_{\text {lat, } 1 \text { (ref) }}=5 \mathrm{mV}$, orange line). In our case the scan size $(l)$ was $20 \mathrm{~nm}$ and the number of pixel/line ( $n_{\text {line }}$ ) was 256 . Due to the definition of the amplitude as half of the peak-to-peak value, the number of pixel over which was smoothed ( $\left.n_{\text {smooth }}\right)$ needs to be devided by two after one pixel was substracted (the pixel at the center position). The determination of the optical invOLS was done by comparing the differences of the lateral amplitudes in nanometers and in volts such as shown in equation (3). Following the explained procedure, we determined a lateral sensitivity of $122 \mathrm{~nm} / \mathrm{V}$. The resulting lateral-oscillation-amplitude setpoints in nanometers corresponding to the images in 
Figure 3 are shown in the center row. Consequently, the invOLS of the lateral oscillation is around eleven times larger compared to the invOLS of the torsional oscillation which we determined in our recent work to be $11 \mathrm{~nm} / \mathrm{V}^{4}$. At first glance this seems to be counterintuitive because the lateral stiffness is higher than the torsional stiffness for the type of cantilever used here (see Supplementary Information for details), which should also result in a higher optical lever sensitivity (lower invOLS) for the lateral oscillation. However, if we take into account that the signal on the photodiode cannot sense the lateral but only the torsional component of the lateral oscillation induced by the tip, it is reasonable to believe that the invOLS is larger for the lateral eigenmode oscillation.

\section{Comparison of torsional and lateral frequency-shift images as a function of the amplitude setpoints}

In order to gain deeper insights into the different oscillation behaviors of the torsional and the lateral eigenmodes, we analyzed several $5 \times 5 \mathrm{~nm}^{2}$ frequency-shift images taken in the AMFlex2-FMLat1-FMFlex3 mode and the AMFlex2-FMTor1-FMFlex3 mode at different lateral- and torsional-eigenmode setpoints. The results are shown in Figure 4. In Figure 4a,e and Figure 4d,h lateral and torsional frequency-shift images $\left(2.5 \times 2.5 \mathrm{~nm}^{2}\right.$ zoom-in) taken at $A_{\text {lat }, 1}=518 \mathrm{pm}(\mathrm{a}), A_{\text {lat }, 1}=3451 \mathrm{pm}(\mathrm{e})$ and $A_{t o r, 1}=158 \mathrm{pm}(\mathrm{d}), A_{t o r, 1}=628 \mathrm{pm}(\mathrm{h})$ are shown exemplarily. The corresponding histograms of the lateral and the torsional frequency-shift images can be seen in Figure $4 \mathrm{~b}$ and $\mathrm{c}$, respectively. The open circles in Figure $4 \mathrm{f}$ and $\mathrm{g}$ show the lateral and torsional frequency shifts at maximum counts and the dashed lines represent the full width at half maximum (FWHM) as a function of the lateral- and torsional-amplitude setpoints, respectively. 

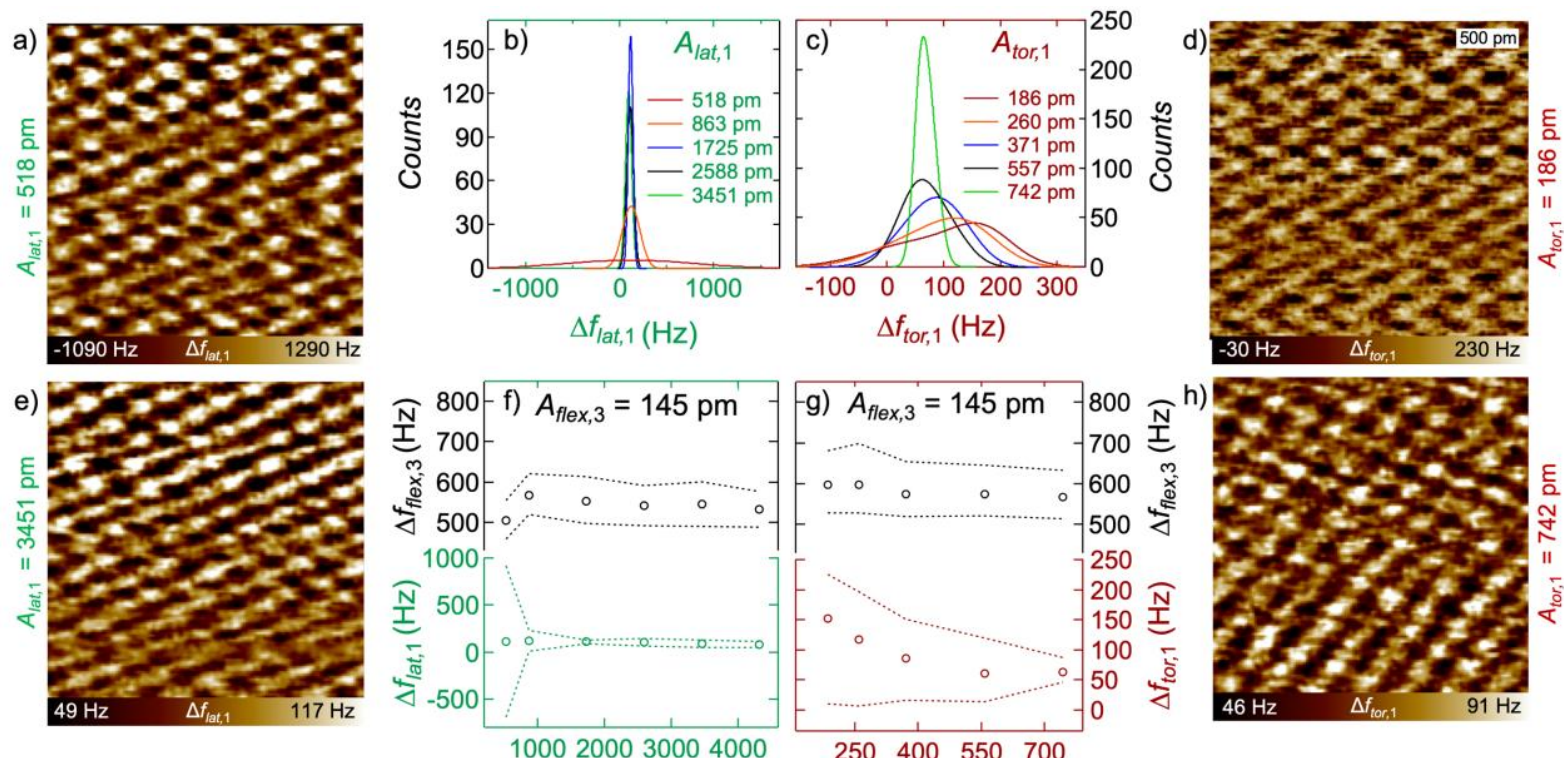

$A_{\text {lat }, 1}(\mathrm{pm})$
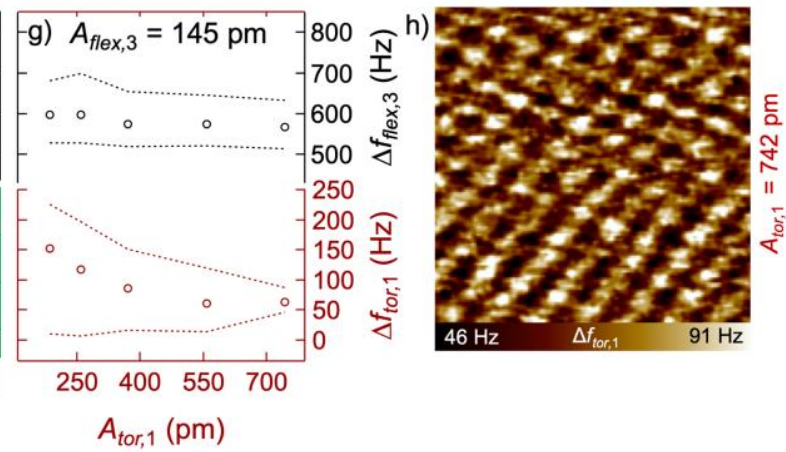

Figure 4: Influence of the lateral- and the torsional-amplitude setpoints on the frequency shifts in AMFlex2-FMLat1-FMFlex3 mode and in AMFlex2-FMTor1-FMFlex3 mode respectively. Frequency-shift images are shown exemplarily taken at $A_{\text {lat }, 1}=518 \mathrm{pm}$ in (a), $A_{\text {lat }, 1}=3451 \mathrm{pm}$ in (e) and $A_{t o r, 1}=158 \mathrm{pm}$ in (d), $A_{t o r, 1}=628 \mathrm{pm}$ in (h). Histograms of lateral (b) and torsional frequency-shift images (c) at different lateral-/torsional-amplitude setpoints. Third-eigenmode flexural and lateral (f)/torsional (g) frequency shifts at maximum counts (open circles) and at FWHM (dotted lines) as a function of the lateral-/torsional-amplitude setpoints at a constant third flexural-eigenmode amplitude of $145 \mathrm{pm}$ and a constant second flexural-eigenmode amplitude of $110 \mathrm{pm}$.

If we compare the frequency-shift images taken in the AMFlex2-FMLat1-FMFlex3 mode in Figure 4a,e with the images taken in the AMFlex2-FMTor1-FMFlex3 mode in Figure 4d,h we observe that atomic contrast was achieved in all images but the range of the frequency-shift values strongly differs. While the lateral and torsional frequency-shift images taken at the higher-amplitude setpoints $\left(A_{\text {lat, } 1}=3451 \mathrm{pm}\right.$ and $\left.A_{t o r, 1}=628 \mathrm{pm}\right)$ shown in Figure $4 \mathrm{e}$ and $\mathrm{h}$ show similar frequency-shift ranges, the frequency-shift images at smaller-amplitude setpoints 
$\left(A_{l a t, 1}=518 \mathrm{pm}\right.$ and $\left.A_{t o r, 1}=158 \mathrm{pm}\right)$ in Figure $4 \mathrm{a}$ and e clearly differ regarding their frequencyshift ranges and the distribution of the values. This becomes even more evident by looking at the histograms corresponding to frequency-shift images taken at five different lateral- (Fig. 4b) and torsional-amplitude (Fig. 4c) setpoints. In general, the full width at half maximum (FWHM) of the peaks increases with decreasing amplitude setpoints (lateral/torsional). The histograms of the lateral frequency-shift images remain symmetric around a center value of approximately $120 \mathrm{~Hz}$, whereas the histograms of the torsional frequency-shift images become asymmetric for torsional-amplitude setpoints smaller than $314 \mathrm{pm}$. As a result, the maxima of the histograms are shifted to more repulsive values. Moreover, the third eigenmode flexural frequency shift is only slightly influenced by the lateral- or torsional-eigenmode amplitude which corroborates the assumption of the independency of the in-plane (lateral/torsional) and the out-of-plane (flexural) eigenmodes (Fig. 4f,g (top graphs)). Interestingly, we observe that although the torsional- and lateral-amplitude setpoints are in most cases substantially larger than the interatomic spacing of the carbon atoms, we can still obtain atomic contrast in the frequencyshift images. To further investigate this phenomenon, we calculated the tip-trajectory resulting from the combined lateral-flexural (Fig. 5a,b) or torsional-flexural (Fig. 5c,d) oscillations of the cantilever according to equations $(\mathrm{S} 1, \mathrm{~S} 2)$ for two different lateral and torsional amplitudes, respectively. 

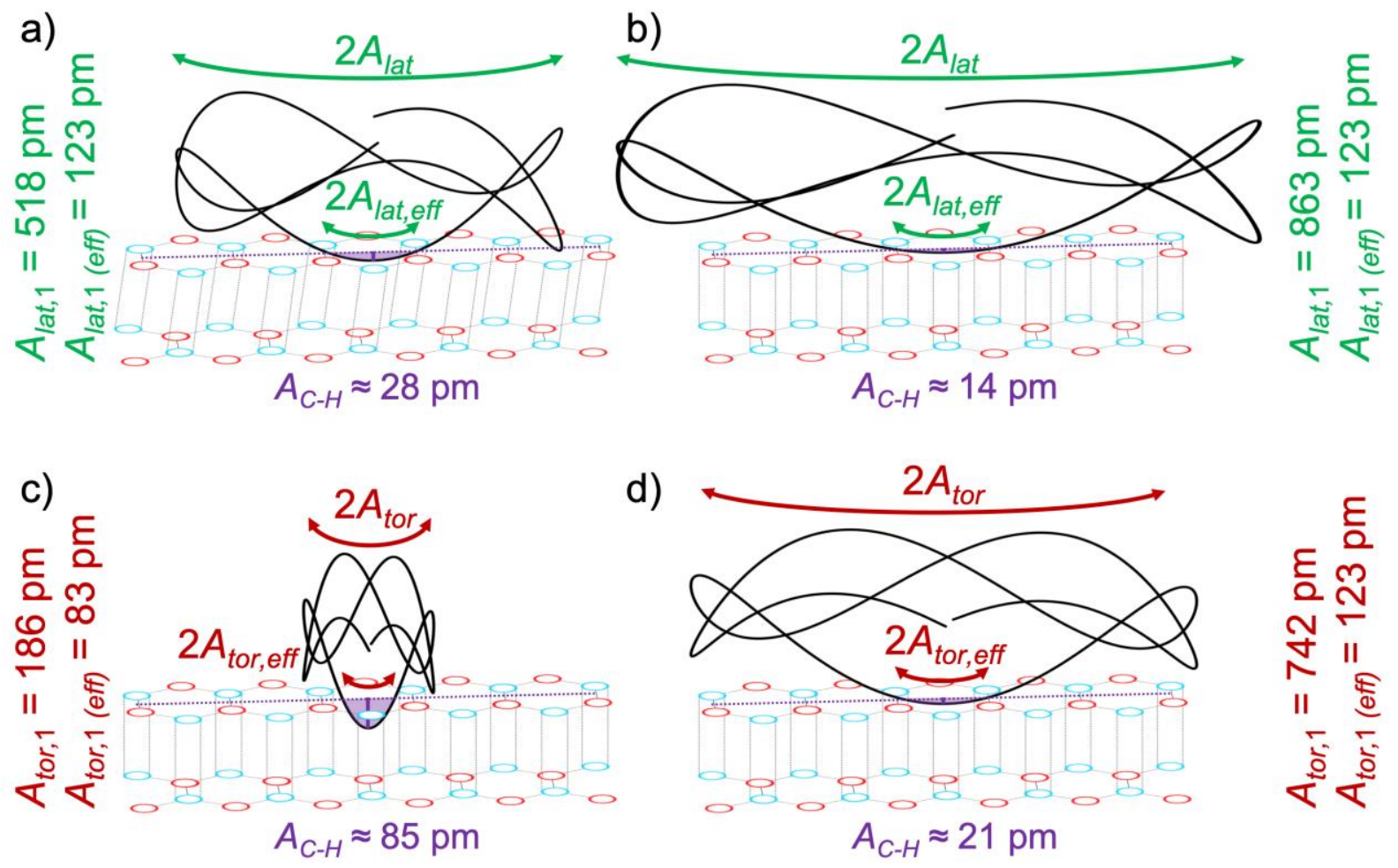

Figure 5: Extract of the calculated tip-trajectory of a cantilever oscillating in the AMFlex2FMLat1-FMFlex3 mode $(\mathrm{a}, \mathrm{b})$ or in the AMFlex2-FMTor1-FMFlex3 mode (c, d) with $A_{f l e x, 2}=110 \mathrm{pm}$ and $A_{f l e x, 3}=145 \mathrm{pm}$. The trajectories are shown for two different torsional- and lateral-oscillation eigenmodes: (a) $A_{l a t, 1}=518 \mathrm{pm}$, (b) $A_{l a t, 1}=863 \mathrm{pm}$, (c) $A_{t o r, 1}=186 \mathrm{pm}$ and (d) $A_{t o r, 1}=742 \mathrm{pm}$. Additionally, the effective amplitudes $\left(A_{\text {lat }, 1(e f f)}\right.$ and $A_{t o r, 1}($ eff $\left.)\right)$ as well as the determined corrugation amplitudes $A_{C-H}$ are shown. Note: The minimum possible distance between two hydrophobic surfaces (tip and sample) of $300 \mathrm{pm}^{29}$ was neglected for the schematic drawing to improve the comprehensibility.

It needs to be mentioned that in Figure 5 only an extract of the full oscillation trajectory is shown. While imaging one position, almost every point in the area spanned by $A_{f l e x, 2}+A_{f l e x, 3}$ and $A_{t o r, 1}$ or $A_{\text {lat }, 1}$, respectively, is reached such as plotted in the Supplementary Information (Fig. S2). From Figure 5 it becomes obvious that the interaction between tip and sample strongly depends on the curvature of the tip. For the situations shown in Figure 5a,b and d we interpret, that the minimum distance from the lower turning point of the tip-trajectory to the intersection of the tip-trajectory and the horizontal line (purple dotted) defined by the 
interatomic spacing between two atoms (here $246 \mathrm{pm}$, due to the tip oscillating perpendicular to the carbon bonds) determines the intend (without distortion of the carbon atoms) of the tip into the carbon ring (vertical purple line). This indent without distortion of carbon atoms corresponds to the corrugation amplitude $\left(A_{C-H}\right)$. Thus, we defined the effective in-plane amplitudes as half of the horizontal distance between two carbon atoms (here: Alat, 1 (eff) $=$ $A_{\text {tor }, 1(e f f)}=123 \mathrm{pm}$ because the tip oscillates perpendicular to the carbon bonds) for the situations shown in Figure 5a,b and d. From the situation shown in Figure 5c we found that if the tip-trajectory shows a higher curvature, the effective amplitude is no longer equivalent to half of the distance between two carbon bonds. This is a result of the maximum possible corrugation amplitude which was reported by Kawai et al. to equal $85 \mathrm{pm}$ in the repulsive regime $^{28}$. Consequently, the effective amplitude is still determined by the intersection between the tip-trajectory and the horizontal line (purple dotted) defined by the interatomic spacing between two atoms, but it becomes smaller than $123 \mathrm{pm}$ due to the limited corrugation amplitude. If we calculate the effective torsional amplitude from the tip-trajectory in Figure 5c, assuming a corrugation amplitude of $85 \mathrm{pm}$, it would be approximately $83 \mathrm{pm}$, which is in line with the $79 \mathrm{pm}$ determined in our recent work ${ }^{4}$. We can conclude that imaging the atomic structure of HOPG in the frequency-shift channel is also possible with in-plane oscillations larger than half of the interatomic spacing, but the resolution in the height images is strongly reduced due to the small corrugation amplitudes. Additionally, it needs to be considered that the frequency-shift images reflect the tip-sample interaction averaged over a few carbon hexagons. This needs to be kept in mind if forces are determined from the frequency-shift images. Nevertheless, due to the formation of plateaus in the torsional and lateral frequency shift values for larger in-plane oscillation amplitudes (see Fig. 4f,g (bottom graph)), we propose that conclusions about different interaction mechanisms, i.e. friction or shear, as a function of the different in-plane oscillation amplitudes can be drawn. 
We point out that the most obvious way of determining the corrugation amplitude would be the analysis of the topography images. Unfortunately, the topography images are strongly influenced by the mean cantilever deflection, so that the corrugation amplitude is not directly accessible ${ }^{4}$.

\section{Calculation of lateral and torsional forces}

From Figure 4, we additionally observed that the full width at half maximum becomes much broader for the smaller lateral-amplitude setpoints compared to the torsional-amplitude setpoints. In order to identify the origin of the differences between torsional and lateral frequency-shift behavior, we calculated the forces from the images taken at $A_{l a t, 1}=518 \mathrm{pm}$ and $A_{\text {tor }, 1}=158 \mathrm{pm}\left(A_{\text {flex }, 2}=110 \mathrm{pm}\right.$ and $\left.A_{\text {flex }, 3}=145 \mathrm{pm}\right)$, respectively. The results are presented in Figure 6. Schemes of the interaction between tip and sample are shown in Figure 6a and d. The lateral and torsional frequency-shift images are shown in Figure $6 \mathrm{~b}$ and e. In Figure $6 \mathrm{c}$ and $\mathrm{f}$ forces calculated from the frequency-shift images in Figures $6 \mathrm{~b}$ and e by using the Fourier method $^{4,30}$ are shown. 
a)
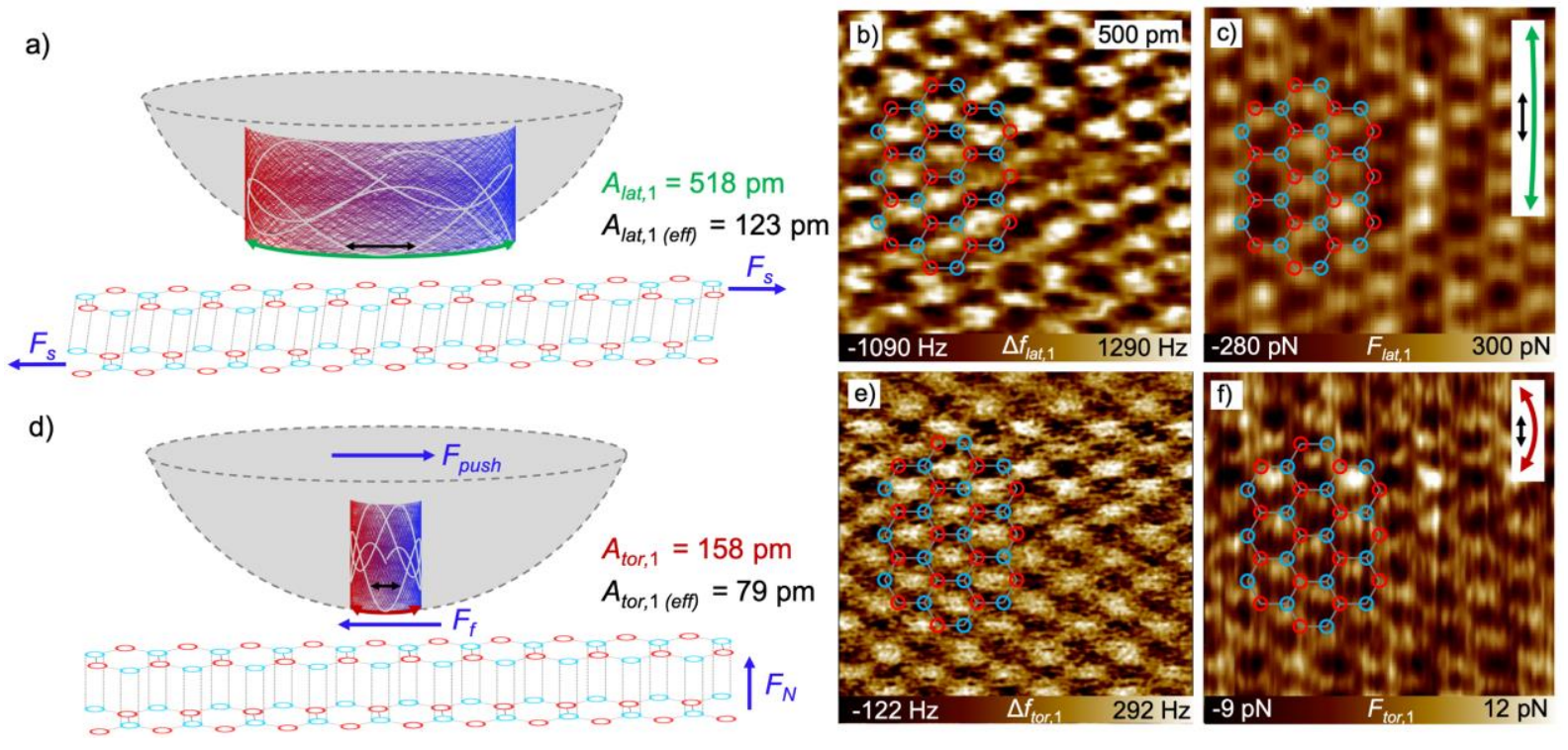

Figure 6: Scheme of the interaction between tip and HOPG surface for (a) a shearing interaction resulting from imaging in the AMFlex2-FMLat1-FMFlex3 mode with $A_{\text {lat }, 1}=518 \mathrm{pm}\left(F_{s}\right.$ : shear force) and (d) a frictional interaction resulting from imaging in the AMFlex2-FMTor1FMFlex 3 mode with $A_{t o r, 1}=158 \mathrm{pm}$, where $F_{N}$ is the normal force acting perpendicular to the surface, $F_{p u s h}$ is the force originating from the movement of the tip in the direction of the inplane oscillation and $F_{f}$ is the frictional force counteracting $F_{p u s h}\left(A_{f l e x, 2}=110 \mathrm{pm}\right.$ and $\left.A_{\text {flex }, 3}=145 \mathrm{pm}\right)$. (b, e) lateral and torsional frequency-shift images. $(\mathrm{c}, \mathrm{f})$ corresponding lateral and torsional force images calculated by the Fourier method and using the effective lateral/torsional amplitudes $A_{\text {lat, } 1 \text { (eff) }}=123 \mathrm{pm}$ and $A_{\text {tor }, 1(e f f)}=79 \mathrm{pm}$.

Obviously, both, the torsional and the lateral force image in Figure $6 \mathrm{c}$ and $\mathrm{f}$ reproduce the hexagonal structure of graphene. It needs to be mentioned that this is only the case if the forces are calculated by using the effective torsional or lateral amplitude. Interestingly, forces determined from the lateral frequency-shift image in Figure $6 \mathrm{~b}$ are around 50 times larger compared to the forces (Figure 6f) calculated from the torsional frequency-shift images in Figure 6e. We propose that this large difference in forces originates partially form the difference in force constants but also/mainly from different types of interaction mechanisms between tip 
and sample, i.e. friction and shear. The lateral frequency shift determined at $518 \mathrm{pm}$ lateral amplitude is assumed to originate from a local shear of the carbon layers relative to each other. We assume this to happen for two reasons: First, the angle under which the tip touches the surface at the respective local position is less steep for the shown lateral oscillation compared to the torsional one. Second, the lateral stiffness of the cantilever is higher compared to the torsional stiffness which might lead to shear for a certain range of lateral oscillation amplitudes whereas torsional amplitudes of the same magnitude result in frictional interaction between tip and sample. In order to verify our assumption about shearing interaction for the constellation shown in Figure 6a-c we calculated the lateral displacement $\Delta x$ of the topmost carbon layer relative to the second one if we assume a shear modulus of $4.18 \mathrm{GPa}$ in $\mathrm{x}$-z-direction ${ }^{31}$. In a simple approximation, the shear modulus $G_{x z}$ can be calculated by

$$
G_{x z}=\frac{F_{l a t} \cdot d}{\Delta x \cdot A_{c}}
$$

where $d$ is the distance between two carbon layers $(334 \mathrm{pm})$ and $A_{c}$ is the contact area between tip and sample which can be calculated according to Hertz from the tip radius $R$ and the indentation $\delta^{32}$

$$
A_{c}=\pi R \delta
$$

In order to determine the indentation $\delta$ which is caused by the tip oscillating over a carbon atom, we used Hertz contact mechanics model ${ }^{32}$

$$
F=\frac{4}{3} E^{*} \sqrt{R \delta^{3}}
$$

where $E^{*}$ is the effective Young's modulus which can be considered here as equal to the Young's modulus $E_{\mathrm{zz}}$ of graphite due to the high stiffness of the diamond-like-carbon tip. The maximum value of $F_{f l e x, 3}$ was calculated in our resent work to be approximately $520 \mathrm{pN}$ for the 
AMFlex2-FMTor1-FMFlex3 mode ${ }^{4}$. Due to the similarity of the third-eigenmode flexural frequency-shift values we chose this value as representative for all the other experiments. By rearranging equation (6) and inserting $E_{\mathrm{zz}}=36.5 \mathrm{GPa}$ as reported by Blakslee et $a l .{ }^{33}$ we determined an indentation of approximately $50 \mathrm{pm}$. Using $\delta=50 \mathrm{pm}$ and rearranging equation (4), we calculated a local displacement of approximately $\Delta x=157 \mathrm{pm}$ between the first and the second graphene layer. This implies a shift by the length of approximately one carbon-carbon bond (142 pm) which seems to be reasonable in distance.

For the tip-trajectory of the torsional oscillation shown in Figure 6d, we observe a very similar oscillatory behavior, however, much more compact in the in-plane direction, resulting from the smaller torsional amplitude. As a consequence, the angle at which the tip approaches and finally touches the surface is much steeper and therefore a negligible shifting of the first carbon layer relative to the second is assumed to take place. We suppose that the torsional forces involved in this mechanism can be interpreted as strongly localized frictional forces which is on the one hand in accordance with the magnitude of frictional forces on graphene determined by Lee $e t$ $a l .{ }^{34}$. On the other hand, the interpretation is in compliance with the observation of direction dependent torsional forces observed in our recent work ${ }^{4}$. Additionally, it needs to be highlighted, that the flexural/torsional oscillation of the cantilever simulated here is symmetric in out-of-plane direction but not in in-plane direction such as schematically shown in Figure S2 of the Supplementary Information. This can explain the shift between topography and frequency-shift images such as shown in our resent work ${ }^{4}$. For the flexural/lateral oscillation the tip-trajectory is overall symmetric in both, in-plane and out-of-plane direction (see Figure S2). Here, no shift between the height and the frequency-shift images could be observed. However, it needs to be mentioned that the resolution in the height images was strongly reduced if imaging was accomplished with the lateral instead of the torsional eigenmode. The effect can be attributed to the shear interaction between tip and sample. If the lateral or the torsional 
amplitudes become larger in size, we observe averaged friction along the surface which might explain the formation of the plateaus in the frequency shifts in Figure $4 \mathrm{f}$ and $\mathrm{g}$ (bottom graph). Here, we also observed that the flexural frequency-shift values and therefore the flexural forces are only slightly influenced by the in-plane oscillation amplitudes. In theory, it should additionally be possible to determine the in-plane Young's moduli $E_{x x}=E_{y y}$ and $E_{x y}$ by using higher torsional or lateral eigenmodes of the cantilever. Unfortunately, it is nontrivial to control these higher eigenmodes and determine their invOLS as well as the corresponding force constants. Nevertheless, this approach could be considered as an alternative method for the determination of the Young's modulus of graphene instead of the frequently used techniques where graphene is spanned over membranes or holes and $E_{x x}$ is calculated from nanoindentation experiments $^{35,36}$. Therefore, we propose that the simultaneous determination of strongly localized in-plane and out-of-plane sample properties such as shear moduli and Young's moduli using multifrequency AFM is feasible if very small amplitudes and adequate force constants are used for the analysis.

\section{Conclusion}

In summary, we demonstrated that performing multifrequency AFM with in-plane and out-ofplane components of the tip-motion has additional potential for atomic resolution imaging of HOPG surfaces. The coupling of the second flexural and the first torsional eigenmode facilitates imaging with atomic contrast at higher amplitude-setpoint ratios, although the quantitative interpretation of the results is challenging if the torsional and the lateral oscillation amplitude are simultaneously excited. In order to estimate the inverse optical lever sensitivity of the lateral cantilever eigenmode, a calibration procedure was proposed which is based on imaging a graphene wrinkle with different lateral amplitude setpoints while oscillating perpendicular to 
the wrinkle and comparing the images with the results of a Savitzky-Golay-filtered reference image. We compared the results from imaging in the AMFlex2-FMLat1-FMFlex3 and the AMFlex2-FMTor1-FMFlex3 mode. Analyzing the tip-trajectories and the in-plane forces resulting from both modes with the respective amplitudes, we proposed that imaging with small lateral amplitudes can result in localized shear of the first graphite layer with respect to the second one with a relative displacement of $157 \mathrm{pm}$. The applied out-of-plane forces caused an indentation of approximately $50 \mathrm{pm}$ of the top graphene layer. Both values are in a reasonable range to propose that the AMFlex2-FMLat1-FMFlex3 method can be used for the simultaneous determination of $E_{x z}=E_{y z}$ and $G_{x z}=G_{y z}$. Imaging with the AMFlex2-FMTor1-FMFlex3 in contrast, promotes rather frictional forces which we mainly attributed to the smaller force constant and the curved tip-trajectory compared to the lateral eigenmode. Based on our findings, we suggest that the use of higher in-plane cantilever eigenmodes or generally stiffer cantilevers can provide a strategy to determine the shear moduli on HOPG and graphene also with torsional eigenmodes. The method carries great potential for future assessment of graphene in nanodevices where local differences in mechanical properties e.g. induced by defects, play a major role. To this end, the in-plane oscillation amplitudes need to be in the range of the interatomic spacings, which is still challenging while imaging in air under ambient conditions.

\section{Materials and Methods}

Sample. The HOPG sample (grade 2 , mosaic spread angle: $0.8 \pm 0.2^{\circ}$ ) was purchased from SPI Supplies (Structure Probe, Inc., West Chester, PA, USA). Cleaving was performed prior to the AFM experiments with adhesive tape in air under ambient conditions to expose a fresh clean surface. 
Cantilevers. Super-sharp cantilevers of the type HiResC $15 / \mathrm{Cr}-\mathrm{Au}$ purchased from Mikromasch (Innovative Solutions Bulgaria Ltd., Sofia, Bulgaria) were used. Although of the same type, the cantilevers showed slightly varying resonance frequencies resulting in "coupled" and "uncoupled" eigenmodes. For the AFM images shown in Figure 2 we used a cantilever with "coupled" second flexural and first torsional eigenmodes as it depicted a close proximity of both resonance frequencies. The resonance frequencies of the used cantilevers were: $f_{0}\left(\right.$ flex,1) $=274 \mathrm{kHz}, f_{0}\left(\right.$ flex,2) $=1.718 \mathrm{MHz}, f_{0}($ tor $)=1.735 \mathrm{MHz}$ and $f_{0}($ lat $)=1.913 \mathrm{MHz}$. For the AFM images shown in Figure 4 and Figure 6 cantilevers with uncoupled eigenmodes were used. The resonance frequencies of the cantilever used for the AMFlex2-FMTor1-FMFlex3 experiments were $\quad f_{0}($ flex, 1$)=266 \mathrm{kHz}, \quad f_{0}\left(f\right.$ lex,2) $=1.667 \mathrm{MHz}, \quad f_{0}(f$ lex,3) $=4.615 \mathrm{MHz}$, $f_{0(t o r)}=1.730 \mathrm{MHz}$ and $f_{0(l a t)}=1.887 \mathrm{MHz}$ and for the AMFlex2-FMLat1-FMFlex3 experiments $\quad f_{0(f l e x, 1)}=263 \mathrm{kHz}, \quad f_{0(\text { flex }, 2)}=1.653 \mathrm{MHz}, \quad f_{0}($ flex,3) $=4.562 \mathrm{MHz}$, $f_{0(t o r)}=1.720 \mathrm{MHz}$ and $f_{0}($ lat $)=1.872 \mathrm{MHz}$. The respective force constants were determined as $k_{\text {flex }, 1}=26 \mathrm{~N} / \mathrm{m}, \quad k_{\text {flex }, 2}=644 \mathrm{~N} / \mathrm{m}, \quad k_{\text {flex }, 3}=3489 \mathrm{~N} / \mathrm{m}, \quad k_{\text {tor }}=472 \mathrm{~N} / \mathrm{m} \quad$ and $\quad k_{\text {lat }}=1440 \mathrm{~N} / \mathrm{m}$ (details are given in the Supplementary Information). The tip radius of the cantilevers was $R=1 \mathrm{~nm}$ according to the manufacturers data sheet.

Environmental conditions. The temperature and the relative humidity in the AFM chamber were not actively controlled, however, imaging conditions remained stable during imaging $\left(22 \pm 2 \%, 26 \pm 2^{\circ} \mathrm{C}\right)$.

AFM setup. A Cypher S atomic force microscope (Asylum Research, Oxford Instruments, Santa Barbara, CA, USA) equipped with a built-in blueDrive photothermal excitation setup for dynamic AFM modes was used for all experiments. The respective frequency shifts (depending on the method used) were tracked with additional phase-locked loops (HF2PLL, Zurich Instruments, Zurich, Switzerland). The drive amplitudes of the frequency-modulated 
eigenmodes were adjusted in order to maintain constant amplitudes by two PID controllers implemented in the same instrument.

Data processing. The topography and the torsional-amplitude error images were first-order flattened to remove any tilt from the images using the Igor Pro v6.36 software (WaveMetrics Inc., Lake Oswego, OR, USA). In order to remove noise from the small-scale images a $3 \times 2$ Gauss filter was applied to all AFM images except for the ones used for the determination of the lateral invOLS (Figure 3). For the calculation of the torsional and the lateral force images in Figure 6 we wrote a Matlab code (MATLAB R2018a, MathWorks Inc., Natick, MA, USA) for the implementation of the Fourier method based on the script provided by Seeholzer et $a l .{ }^{4,30}$. The calculated force images were smoothed with a Savitzky-Golay filter over nine points with a first-order polynomial.

\section{Data availability}

All data will be made available from the authors upon reasonable request.

\section{References}

1 Wang, J., Zhang, D. \& Zhou, B. Achieving an Ohmic contact in graphene-based van der Waals heterostructures by intrinsic defects and the inner polarized electric field of Janus AlGaSSe. New Journal of Chemistry 45, 21178-21187, doi:10.1039/D1NJ03861C (2021).

2 Heerema, S. J. \& Dekker, C. Graphene nanodevices for DNA sequencing. Nature Nanotechnology 11, 127-136, doi:10.1038/nnano.2015.307 (2016).

3 Garcia, R. \& Herruzo, E. T. The emergence of multifrequency force microscopy. Nature Nanotechnology 7, 217-226, doi:10.1038/nnano.2012.38 (2012). 
4 Eichhorn, A. L. \& Dietz, C. Simultaneous Deconvolution of In-Plane and Out-of-Plane Forces of HOPG at the Atomic Scale under Ambient Conditions by Multifrequency Atomic Force Microscopy. Advanced Materials Interfaces 8, doi:10.1002/admi.202101288 (2021).

5 Uhlig, M. R., Martin-Jimenez, D. \& Garcia, R. Atomic-scale mapping of hydrophobic layers on graphene and few-layer MoS2 and WSe2 in water. Nature Communications 10, 2606, doi:10.1038/s41467-019-10740-w (2019).

6 Baykara, M. Z. et al. Exploring atomic-scale lateral forces in the attractive regime: a case study on graphite (0001). Nanotechnology 23, 405703, doi:10.1088/09574484/23/40/405703 (2012)

7 Sader, J. E. \& Jarvis, S. P. Accurate formulas for interaction force and energy in frequency modulation force spectroscopy. Applied Physics Letters 84, 1801-1803, doi:10.1063/1.1667267 (2004).

8 Giessibl, F. J. A direct method to calculate tip-sample forces from frequency shifts in frequency-modulation atomic force microscopy. Applied Physics Letters 78, 123-125, doi:10.1063/1.1335546(2000).

9 Kawai, S. et al. Ultrasensitive detection of lateral atomic-scale interactions on graphite (0001) via bimodal dynamic force measurements. Physical Review B 81, 085420, doi:10.1103/PhysRevB.81.085420 (2010).

10 Dietz, C. Sensing in-plane nanomechanical surface and sub-surface properties of polymers: local shear stress as function of the indentation depth. Nanoscale 10, 460468, doi:10.1039/c7nr07147g (2018).

11 Pini, V., Tiribilli, B., Gambi, C. M. C. \& Vassalli, M. Dynamical characterization of vibrating AFM cantilevers forced by photothermal excitation. Physical Review B $\mathbf{8 1}$, 054302, doi:10.1103/PhysRevB.81.054302 (2010). 
12 Kiracofe, D., Kobayashi, K., Labuda, A., Raman, A. \& Yamada, H. High efficiency laser photothermal excitation of microcantilever vibrations in air and liquids. Review of Scientific Instruments 82, 013702, doi:10.1063/1.3518965 (2011).

13 Meier, T., Eslami, B. \& Solares, S. D. Multifrequency force microscopy using flexural and torsional modes by photothermal excitation in liquid: atomic resolution imaging of calcite (10(1)over-bar4). Nanotechnology 27, 085702, doi:10.1088/09574484/27/8/085702 (2016).

14 Ding, R.-F., Yang, C.-W., Huang, K.-Y. \& Hwang, I.-S. High-sensitivity imaging with lateral resonance mode atomic force microscopy. Nanoscale 8, 18421-18427, doi:10.1039/C6NR04151E (2016).

15 Mullin, N. \& Hobbs, J. K. A non-contact, thermal noise based method for the calibration of lateral deflection sensitivity in atomic force microscopy. Review of Scientific Instruments 85, 113703, doi:10.1063/1.4901221 (2014).

16 Ogletree, D. F., Carpick, R. W. \& Salmeron, M. Calibration of frictional forces in atomic force microscopy. Review of Scientific Instruments 67, 3298-3306, doi:10.1063/1.1147411 (1996).

17 Varenberg, M., Etsion, I. \& Halperin, G. An improved wedge calibration method for lateral force in atomic force microscopy. Review of Scientific Instruments 74, 33623367, doi:10.1063/1.1584082 (2003).

18 Zhang, G., Li, P., Wei, D., Hu, K. \& Qiu, X. Lateral force calibration for atomic force microscope cantilevers using a suspended nanowire. Nanotechnology 31, 475703, doi:10.1088/1361-6528/abae32 (2020).

19 Kawai, S., Kitamura, S.-i., Kobayashi, D., Meguro, S. \& Kawakatsu, H. An ultrasmall amplitude operation of dynamic force microscopy with second flexural mode. Applied Physics Letters 86, 193107, doi:10.1063/1.1923200 (2005). 
20 Korolkov, V. V. et al. Supramolecular networks stabilise and functionalise black phosphorus. Nature Communications 8, 1385, doi:10.1038/s41467-017-01797-6 (2017).

21 Santos, S. et al. Stability, resolution, and ultra-low wear amplitude modulation atomic force microscopy of DNA: Small amplitude small set-point imaging. Applied Physics Letters 103, 063702, doi:10.1063/1.4817906 (2013).

22 Lai, C. Y., Santos, S. \& Chiesa, M. Systematic Multidimensional Quantification of Nanoscale Systems From Bimodal Atomic Force Microscopy Data. Acs Nano 10, 62656272, doi:10.1021/acsnano.6b02455 (2016).

23 Santos, S., Olukan, T. A., Lai, C.-Y. \& Chiesa, M. Hydration Dynamics and the Future of Small-Amplitude AFM Imaging in Air. Molecules 26, doi:10.3390/molecules26237083 (2021).

24 Benaglia, S., Amo, C. A. \& Garcia, R. Fast, quantitative and high resolution mapping of viscoelastic properties with bimodal AFM. Nanoscale 11, 15289-15297, doi:10.1039/c9nr04396a (2019).

25 Kawai, S. et al. Time-averaged cantilever deflection in dynamic force spectroscopy. Physical Review B 80, 085422, doi:10.1103/PhysRevB.80.085422 (2009).

26 Yalcin, S. E., Legg, B. A., Yeşilbaş, M., Malvankar, N. S. \& Boily, J.-F. Direct observation of anisotropic growth of water films on minerals driven by defects and surface tension. Science Advances 6, eaaz9708, doi:10.1126/sciadv.aaz9708 (2020).

27 Abooalizadeh, Z., Sudak, L. J. \& Egberts, P. Nanoscale spatial mapping of mechanical properties through dynamic atomic force microscopy. Beilstein journal of nanotechnology 10, 1332-1347, doi:10.3762/bjnano.10.132 (2019).

28 Kawai, S. \& Kawakatsu, H. Surface-relaxation-induced giant corrugation on graphite (0001). Physical Review B 79, 115440, doi:10.1103/PhysRevB.79.115440 (2009). 
29 Zhang, Z., Ryu, S., Ahn, Y. \& Jang, J. Molecular features of hydration layers probed by atomic force microscopy. Physical Chemistry Chemical Physics 20, 30492-30501, doi:10.1039/C8CP06126B (2018).

30 Seeholzer, T., Gretz, O., Giessibl, F. J. \& Weymouth, A. J. A Fourier method for estimating potential energy and lateral forces from frequency-modulation lateral force microscopy data. New Journal of Physics 21, 083007, doi:10.1088/1367-2630/ab3353 (2019).

31 Michel, K. H. \& Verberck, B. Theory of the elastic constants of graphite and graphene. Physica Status Solidi B-Basic Solid State Physics 245, 2177-2180, doi:10.1002/pssb.200879604 (2008).

32 Hertz, H. Ueber die Berührung fester elastischer Körper. 1882, 156-171, doi:10.1515/crll.1882.92.156 (1882).

33 Blakslee, O. L., Proctor, D. G., Seldin, E. J., Spence, G. B. \& Weng, T. Elastic Constants of Compression-Annealed Pyrolytic Graphite. Journal of Applied Physics 41, 33733382, doi:10.1063/1.1659428 (1970).

34 Lee, H., Lee, N., Seo, Y., Eom, J. \& Lee, S. Comparison of frictional forces on graphene and graphite. Nanotechnology 20, 325701, doi:10.1088/0957-4484/20/32/325701 (2009).

35 Frank, I. W., Tanenbaum, D. M., Van der Zande, A. M. \& McEuen, P. L. Mechanical properties of suspended graphene sheets. Journal of Vacuum Science \& Technology B 25, 2558-2561, doi:10.1116/1.2789446 (2007).

36 Zandiatashbar, A. et al. Effect of defects on the intrinsic strength and stiffness of graphene. Nature Communications 5, 3186, doi:10.1038/ncomms4186 (2014). 


\section{Acknowledgements}

The authors thank the Deutsche Forschungsgemeinschaft (Project number 407750697) for financial support.

\section{Author contributions}

A.L.E. extended the AFM measurement method, performed the experiments, conducted data analysis and wrote the manuscript. A.L.E. and C. D. designed the experiment. C.D. proposed the basic idea for the method, discussed the results, and revised the manuscript.

\section{Additional information}

Supplementary Information is available online or from the author.

Competing interests: The authors declare no competing interests. 


\section{Supplementary Files}

This is a list of supplementary files associated with this preprint. Click to download.

- 220203SIPaperFlexTorLatScientificreportsfinal.pdf 